\title{
Enhanced stability of laser wakefield acceleration using dielectric capillary tubes
}

\author{
M. Hansson, ${ }^{*}$ L. Senje, A. Persson, O. Lundh, and C.-G. Wahlström \\ Department of Physics, Lund University, P.O. Box 118, S-22100 Lund, Sweden \\ F. G. Desforges, J. Ju, T. L. Audet, and B. Cros \\ Laboratoire de Physique des Gaz et des Plasmas, CNRS-Université Paris-Sud 11, 91405 Orsay, France \\ S. Dobosz Dufrénoy, and P. Monot \\ Service des Photons, Atomes et Molécules, CEA Saclay, 91191 Gif-sur-Yvette, France
}

(Received 25 October 2013; published 17 March 2014)

\begin{abstract}
The stability of beams of laser wakefield accelerated electrons in dielectric capillary tubes is experimentally investigated. These beams are found to be more stable in charge and pointing than the corresponding beams of electrons accelerated in a gas jet. Electron beams with an average charge of $43 \mathrm{pC}$ and a standard deviation of $14 \%$ are generated. The fluctuations in charge are partly correlated to fluctuations in laser pulse energy. The pointing scatter of the electron beams is measured to be as low as $0.8 \mathrm{mrad}$ (rms). High laser beam pointing stability improved the stability of the electron beams.
\end{abstract}

Laser wakefield accelerators, first proposed in 1979 [1], appear promising as sources of highly relativistic electrons and associated X-ray radiation, in particular since experiments have demonstrated the possibility of generating highquality quasimonoenergetic pulses of electrons [2-4].

Laser wakefield accelerators benefit from the much higher electric field that can be sustained in electron plasma waves compared to conventional accelerators, and thus hold promise for size and cost reduction. The beams of laser wakefield accelerated electrons have properties that are complementary to those of conventional accelerators, and thus open doors to novel applications. These sources therefore attract worldwide interest from many different areas of science, technology, and medicine, e.g., X-ray freeelectron lasers, high-energy particle physics, and oncology. However, laser-plasma accelerators are still at their early stage of development and are hampered by, in particular, limited reproducibility and stability, restricting their use in applications.

Much current research in this field is focused on increasing the maximum energy of the electrons, for example by extending the acceleration length through the use of different laser waveguiding structures [5,6], or by using multiple accelerator stages [7]. Large efforts are also made to control the injection of electrons into the

\footnotetext{
*martin.hansson@fysik.lth.se
}

Published by the American Physical Society under the terms of the Creative Commons Attribution 3.0 License. Further distribution of this work must maintain attribution to the author(s) and the published article's title, journal citation, and DOI. accelerating structure to increase the quality of the electron pulses and to gain better control of the mechanism.

In this paper we report on a study of the stability of beams of laser-plasma accelerated electrons using dielectric capillary tubes as laser waveguides. This type of waveguide [8] has three main advantages: (i) The plasma density inside the tubes can be arbitrarily low, as the laser beam is then guided purely by reflection from the tube walls [6]. The absence of a minimum density requirement for guiding [9] makes the capillary tube relevant for laser wakefield acceleration over long distance since the maximum achievable electron energy scales with the inverse electron density assuming the process is limited by dephasing [1]. (ii) When the laser focal spot is smaller than the matched focal spot for coupling to the capillary tube, and for laser power larger than the critical power for self-focusing, the laser energy outside the main peak of the focal spot can be reflected from the tube wall back to the laser axis, which helps to sustain relativistic self-focusing and laser guiding over a longer distance than in a gas jet or in a gas cell with similar plasma density $[9,10]$. (iii) the capillary tube provides a shock-free gas medium [11]. In this study electrons from the background plasma are trapped and accelerated in the so-called bubble regime of laser plasma acceleration. This mechanism is simple to achieve experimentally, and has been observed by numerous groups in gas jet, gas cell, or waveguides. Using hydrogen filled capillary tubes, we have performed a statistical analysis of the stability of the parameters of the electrons accelerated by a laser beam which pointing position is stabilized in the focal plane.

The experiments were conducted at the Lund Laser Centre, using a Ti:Sapphire based multi-terawatt laser 


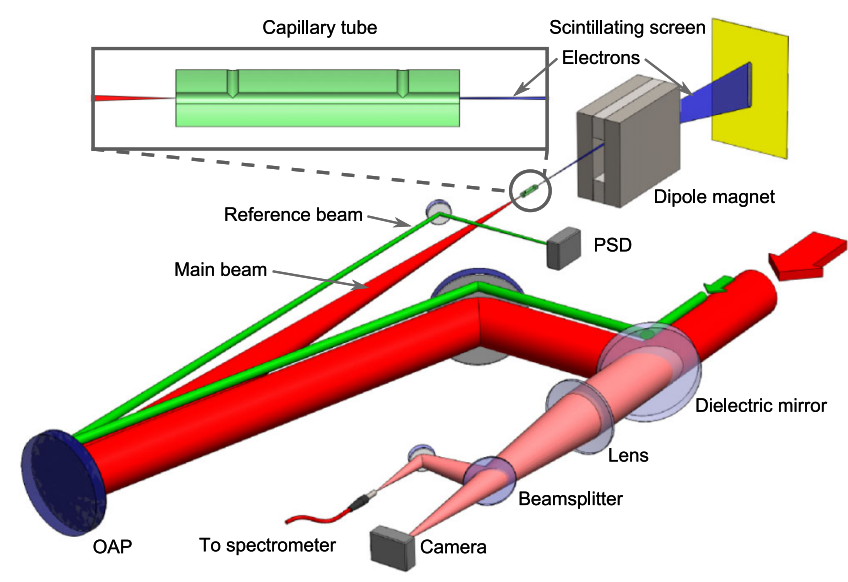

FIG. 1. Sketch of the experimental setup. The main laser pulse (red) is focused by an off-axis parabolic mirror (OAP) into a dielectric capillary tube (or a gas jet) filled with hydrogen gas. The accelerated electrons are dispersed by a dipole magnet before reaching a scintillating screen. The leakage from a dielectric mirror in the beam path is used to record spatial and spectral intensity distributions of every laser pulse. A continuous reference beam (marked in green for clarity, but in reality of the same wavelength as the main beam) is used in combination with a position sensitive detector (PSD) for feedback to a system for active pointing stabilization.

system. A sketch of the experimental setup is shown in Fig. 1. Typically, the laser was set to deliver pulses with energy of $750 \mathrm{~mJ}$ on target with a pulse duration of $40 \mathrm{fs}$ at a center wavelength of $800 \mathrm{~nm}$, giving a peak power of $18 \mathrm{TW}$. The laser beam was focused by an $f=76 \mathrm{~cm}$ offaxis parabolic mirror. Using a closed looped optimization system, consisting of a wavefront sensor and a deformable mirror in the beam path, a focal spot with a FWHM of $19 \mu \mathrm{m}$, close to the diffraction limit (Strehl ratio $\geq 0.95$ ), was achieved. An estimated peak intensity of $4.4 \times 10^{18} \mathrm{~W} / \mathrm{cm}^{2}$ was thus achieved in the focus of the beam, corresponding to a peak normalized vector potential of $a_{0}=1.4$.

An active system for stabilization of the transverse position of the focus [12] was used to minimize laser pointing errors. This system uses an analog position sensitive device to measure the position of the focus from a reference beam, and a large $(100 \mathrm{~mm})$ piezoelectric actuated mirror in the beam path to steer the beam. With this system a short-term scatter of the focal spot position with a rms distance from the average position of $\approx 4 \mu \mathrm{rad}$ was achieved, while any long-term drift of the transverse focal position was essentially eliminated.

The laser pulses were focused one millimeter inside different glass capillary tubes, with diameters in the range 76 to $254 \mu \mathrm{m}$ and lengths in the range 8 to $20 \mathrm{~mm}$, carefully aligned to the laser beam axis [9]. The capillaries were filled with hydrogen gas from a reservoir with variable backing pressure, $35 \mathrm{~ms}$ before each laser pulse. The resulting molecular density of the gas inside each capillary was deducted from interferometric studies [13] performed off-line. The gas inside the capillary tubes became fully ionized into a plasma by the leading edge of the laser pulse. Alternatively, a gas nozzle could be moved under vacuum to replace the capillary tube to create a cylindrical jet of hydrogen gas with a diameter of $3 \mathrm{~mm}$ at the laser focus for comparison with capillary tubes.

The accelerated electrons were observed on a scintillating screen (Kodak Lanex Regular), using a 16-bit CCD camera. This way the electron beam profile and pointing could be studied. In addition, a $120 \mathrm{~mm}$ long rectangular dipole magnet with a peak magnetic field of $0.7 \mathrm{~T}$ could be inserted into the electron beam path to disperse the electrons according to energy and thus allow the energy spectrum of the electrons to be determined. The energy axis of the spectra was absolutely calibrated by numerically tracing electrons through a map of the measured magnetic field. The physical boundaries of the dipole magnet set a lower limit on the energy of the electrons that could be observed to $\approx 40 \mathrm{MeV}$ taking into account the electron beam divergence. The energy resolution of the spectrometer is decreasing with increasing energy. For the divergence of the electron beams observed in this experiment, the energy resolution is $\approx 8 \%$ at $80 \mathrm{MeV}$. The total amount of charge impacting the scintillating screen was determined by integrating the images and using published calibration factors [14] for the scintillating screen.

Furthermore, the laser pulses were sampled, using the leakage through a dielectric mirror in the laser beam path, and focused using an achromatic lens (see Fig. 1). An image of this focus was acquired at each shot, which provided an absolutely calibrated measurement of the laser pulse energy on target and also an estimate of the scatter of the laser focus position and size. In addition, the spectral intensity distribution of each laser pulse was acquired using an optical spectrometer. Neglecting shot-to-shot fluctuations in spectral phase, these spectral intensity variations allow an estimate of shot-to-shot variations in pulse duration to be made through an inverse Fourier transform.

The data presented in Fig. 2 were acquired during a sequence of 130 consecutive pulses with identical settings. The delay between pulses was $30 \mathrm{~s}$ in order to allow the pumps to evacuate the target vacuum chamber from the gas load. Thus, these data points were acquired over $65 \mathrm{~min}$. The measured pulse energy, shown in Fig. 2(a) as a function of the shot number, indicates a slight drift toward lower energy during the sequence of shots. However, the standard deviation of the laser pulse energy is only $1.9 \%$. The estimated laser pulse duration showed similar stability, with a standard deviation of $1.9 \%$, but without any drift. After the first 90 pulses, marked in red in the figures, the active pointing stabilization system was turned off, marked in blue in the figures. As expected, no change in stability of laser energy or pulse duration was observed when turning off the active pointing stabilization system. 


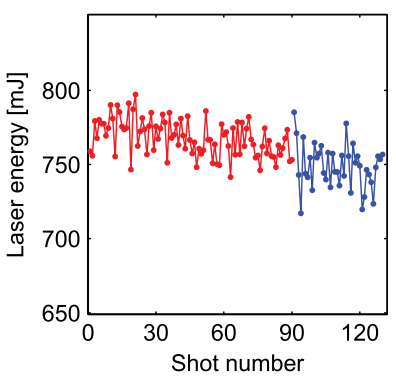

(a)

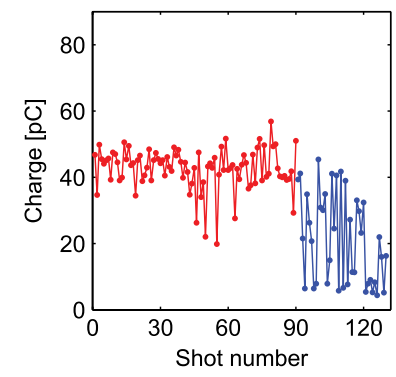

(b)
FIG. 2. Laser pulse energy delivered to the entrance of a $20 \mathrm{~mm}$ long, $152 \mu \mathrm{m}$ diameter capillary tube (a) and the corresponding accelerated charge with an energy above $40 \mathrm{MeV}$ (b) as a function of shot number. The active stabilization system is on during the first 90 pulses, and thereafter off. The stability of the electron bunch charge is significantly decreased by turning this system off.

During this sequence, the laser pulses were focused into a $20 \mathrm{~mm}$ long dielectric capillary tube with a diameter of $152 \mu \mathrm{m}$ filled with hydrogen gas at a backing pressure of 340 mbar, resulting in a fully ionized plasma density of $1.3 \times 10^{19} \mathrm{~cm}^{-3}$. At this plasma electron density and the given peak power, the ratio between the peak power and the critical power for relativistic self-focusing $[15,16]$ is $P / P_{c} \approx 7$. Under these experimental conditions, the laser pulse is expected to self-focus soon after entering the plasma, resulting in an increased peak intensity. The laser pulse expels electrons from regions of high intensity, leading to a bubble structure following the laser pulse, in which electrons are self-injected at multiple locations along the propagation through the plasma $[10,17]$. The density threshold for injection of electrons into the accelerating structure was experimentally determined to be $\approx 0.85 \times 10^{19} \mathrm{~cm}^{-3}$.

The amount of charge in the resulting electron beams, with energy above $40 \mathrm{MeV}$, is shown in Fig. 2(b) as a function of the shot number. The electron beam parameters show larger fluctuations than the laser pulse parameters. Immediately after the active pointing system is turned off, the quality and stability of the electron beams degrade significantly and after $\sim 25$ shots the capillary tube is damaged beyond being operational. This shows that controlling the scatter and drift of the laser focal spot is crucially important when accelerating electrons in dielectric capillary tubes using laser pulses of high intensity and energy. The active stabilization system employed in the present study enabled long sequences of data collection and an enhanced endurance of the capillaries.

The average charge for the first 90 shots in the sequence was determined to $43 \mathrm{pC}$ with a standard deviation of only $14 \%$. This small spread in charge was found to be typical also for dielectric capillary tubes of different dimension after similar optimization. For example, in another sequence the charge of electrons, with energy above $40 \mathrm{MeV}$, accelerated inside a capillary tube with a larger diameter, $254 \mu \mathrm{m}$, and a shorter length, $10 \mathrm{~mm}$, was measured to be higher, $107 \mathrm{pC}$, while the standard deviation was still only $18 \%$. We did not observe any significant correlation between the amount of accelerated charge and the dimensions of the capillary tube. The difference in amount of accelerated charge between the different series of data reported here can instead be attributed to small differences in experimental parameters, such as laser energy and plasma density, that affect the amount of accelerated charge $[17,18]$. The experimental parameters were optimized for best stability before acquiring each series of data, which resulted in slightly different values of these parameters.

The stability in charge using the capillary tube is very good compared to the results achieved when, in the same setup and with identical laser parameters, the capillary tube is replaced with a $3 \mathrm{~mm}$ gas jet as a target, which showed a standard deviation in charge of $55 \%$, with an average value of $68 \mathrm{pC}$. The charge stability of beams accelerated in capillary tubes is very good also when compared to published studies on laser wakefield acceleration in gas jets and gas cells showing stable beams $[11,19,20]$. The average value and stability of measured parameters of the beams of electrons accelerated in dielectric capillary tubes of different sizes, and in a gas jet, are summarized in Table I.

The energy stability of the accelerated electron beams is shown in Fig. 3. False color images of traces of the dispersed electrons on the scintillating screen, from 15 consecutive laser shots (number 25 to 39 in Fig. 2), are shown in Fig. 3(a) using the same color scale for all images. The electron energy spectra in this study show continuous energy distribution, as shown in Fig. 3(b), rather than

TABLE I. Summary of electron beam stability parameters acquired in three series using capillaries of different diameter $(\varnothing)$ and lengths $(l)(\mathrm{A}-\mathrm{C})$ in comparison with one series of data acquired using gas jet. The stabilities of the electron beams were studied using the same regime of acceleration. Before acquiring each series of data, the experimental conditions were optimized for best stability, yielding slightly different values of, e.g., backing pressure and laser intensity. The average and standard deviation of charge $(Q)$ corresponds to electrons with an energy above $40 \mathrm{MeV}$ measured with the dispersing dipole magnet in the electron beam path. The divergence $(\theta)$ and the RMS pointing stability $(\phi)$ was measured without the dispersing dipole in the electron beam path.

\begin{tabular}{lrrrc}
\hline \hline Parameter & A & B & C & Gas jet \\
\hline$\varnothing[\mu \mathrm{m}]$ & 152 & 178 & 254 & $\ldots$ \\
$l[\mathrm{~mm}]$ & 20 & 10 & 10 & 3 \\
$\langle Q\rangle[\mathrm{pC}]$ & 43 & 88 & 107 & 68 \\
std $(Q)[\%]$ & 14 & 14 & 18 & 55 \\
$\langle\theta\rangle[\mathrm{mrad}]$ & 11 & 10 & 10 & 14 \\
std $(\theta)[\%]$ & 13 & 14 & 11 & 64 \\
$\langle\phi\rangle[\mathrm{mrad}]$ & 1.2 & $\ldots$ & 0.8 & 4.4 \\
\hline \hline
\end{tabular}




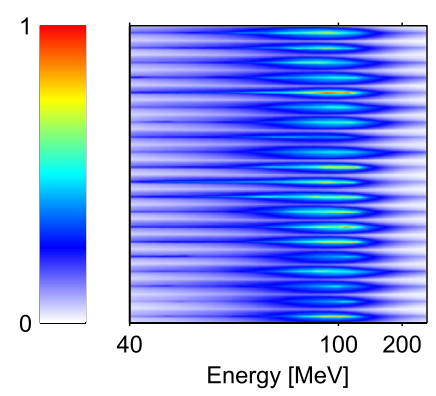

(a)

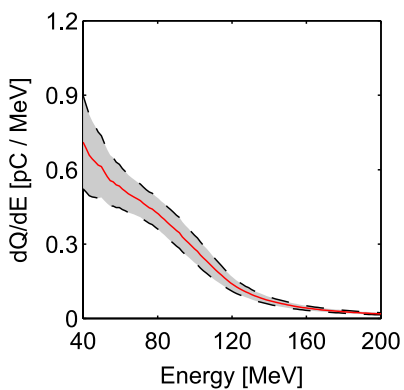

(b)

FIG. 3. False color images of the dispersed electrons on the scintillating screen from 15 consecutive shots are shown in (a), all using the same color scale. The average electron spectrum of all shots in the sequence is plotted as a solid red line in (b). The shaded area, bounded by dashed lines, shows the corresponding standard deviation from the average spectrum. Over the full energy range, the maximum standard deviation from the average spectrum is $27 \%$.

features of peaks, indicating that the acceleration process is dominated by injection at multiple locations in space and time [10,17]. Previous studies [9] report on electron beams from dielectric capillary tubes with quasimonoenergetic energy spectra, but with lower charge, when experimental parameters were instead optimized in order to achieve selfinjection in a well-localized phase-space volume and to avoid dephasing. The parameters of the present study were selected for their capability to produce, on every shot, an accelerated beam with a significant amount of charge, interesting for example for the study of X-ray generation or other applications.

The energy spectrum of the beams of accelerated electrons in dielectric capillary tubes are reproducible, as indicated by Fig. 3. The standard deviation from the average spectrum is less than $27 \%$ over the full measured energy range. This standard deviation is below $15 \%$ for energies between 65 and $80 \mathrm{MeV}$ and has a minimum of $14 \%$ at $72 \mathrm{MeV}$.

The dependence of the amount of accelerated charge, with an energy above $40 \mathrm{MeV}$, on the laser pulse energy on target is shown in Fig. 4(a). This figure shows a clear dependence between the charge and laser pulse energy. This behavior is expected since higher laser energy for the same pulse parameters implies a higher probability of selfinjection due to the higher amplitude of the plasma wave [18]. The data points are scattered along the solid line, which is fitted from the experimental data, and indicates that the stability in charge can be improved by a better stability in laser pulse energy. Although a certain amount of fluctuations in beam charge is clearly due to variations in laser pulse energy, these variations account only for part of the total charge fluctuations within a given series. However, the figure shows that small variations in laser energy can explain the observed difference in accelerated charge between different series.
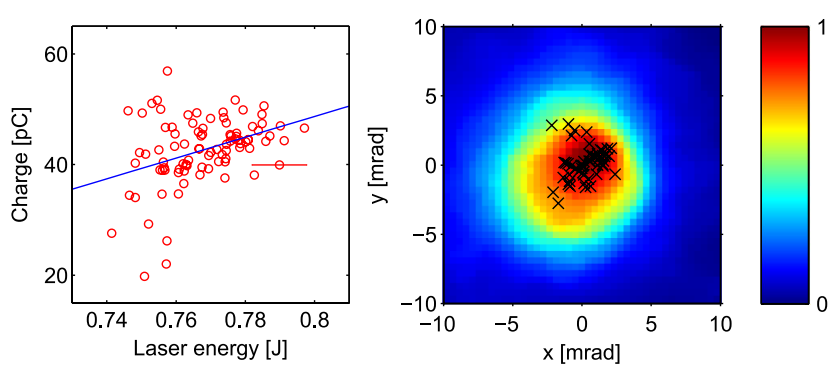

FIG. 4. In (a), the charge of the electrons in the beam, with energy above $\sim 40 \mathrm{MeV}$, is plotted as a function of laser pulse energy for the first 90 shots in Fig. 2, with the active pointing stabilization system on. The estimated measurement error in laser energy of $1 \%$ is marked as a solid red line only in one data point for clarity. This figure shows a clear correlation to laser energy. In (b), a typical beam profile is shown together with a scatter map of the centroids of a sequence of 30 shots with the pointing stabilization system on.

We could not find any significant correlation between the electron beam parameters and intrinsic variations in focal spot size or width of the laser pulse spectrum. Although this might be due to insufficient accuracy of the measurement, it can also be due to the nonlinear laser pulse evolution in the plasma, such as self-phase modulation and relativistic selffocusing. Thus, fluctuations in laser pulse duration and spot size after propagation in plasma are not necessarily the same as the measured initial values.

A typical electron beam charge distribution in the transverse plane is shown in Fig. 4(b) acquired during a sequence of 30 shots using the same setup as above, but without the dispersing dipole magnet in the electron beam path. The average divergence (FWHM) for these shots was measured to $11 \mathrm{mrad}$ with a standard deviation of $13 \%$. This divergence is larger than the opening angle of the capillary tube as viewed from the entrance plane, which is $7.6 \mathrm{mrad}$. Similar divergence of the electron beam was measured on electron beams accelerated inside capillaries tubes of other dimensions as shown in Table I, which suggests that the beam is not limited by the aperture of the capillary tube. The divergence of the beams of electrons accelerated inside the capillary tubes is also similar to divergence of the beams accelerated inside a gas jet (see Table I).

The pointing scatter of the electron beams accelerated inside the capillary with diameter $152 \mu \mathrm{m}$ and length of $10 \mathrm{~mm}$ is also shown in Fig. 4(b), where the center of each beam profile is marked by a cross. The rms distance from the average beam position is only $1.2 \mathrm{mrad}$ for this sequence. This is significantly less than the corresponding value of $4.4 \mathrm{mrad}$ measured for the beams of electrons accelerated inside a gas jet during this experiment. Small pointing scatter was observed also using capillaries of other dimensions. For example, during a sequence of 100 pulses in a capillary with a diameter of $254 \mu \mathrm{m}$ and a length of 
$10 \mathrm{~mm}$, the rms pointing scatter was as low as $0.8 \mathrm{mrad}$ and no shot deviated more than $2.7 \mathrm{mrad}$ from the average beam position.

The beams of electrons accelerated during this experiment have been shown to be very stable in charge, divergence, and pointing. The stable and uniform gas density distribution, which is also shockfree, that can be achieved in a dielectric capillary tube, or a gas cell of similar dimensions, is expected to contribute to this stability of the resulting electron beams.

In conclusion, we have shown an enhanced stability of beams of electrons accelerated in dielectric capillary tubes compared to electrons accelerated using a gas jet as target. Very good laser beam pointing stability has been shown to be necessary in order to carry out the studies reported in this paper. In the present study, such stability was achieved using an active beam pointing stabilization system. The measured beam charge variations are shown to be partly due to laser pulse energy fluctuations. The stability of the electron beam parameters appears to be similar for the different sizes of capillary tubes used in this experiment. The enhanced stability of laser wakefield acceleration using dielectric capillary tubes indicates a path toward applications of these electron beams.

We acknowledge the support of the Swedish Research Council, the Knut and Alice Wallenberg Foundation, the Swedish Foundation for Strategic Research, the Lund University X-ray Centre (LUXC), Laserlab-Europe/ CHARPAC (Grant Agreement No. 284464, EC's 7th Framework Programme) and EuCARD2/ANAC2 (Grant Agreement No. 312453, EC's 7th Framework Programme). J. Ju acknowledges financial support from the Chinese Scholarship Council (CSC). This project has benefited from financial support from ARC.

[1] T. Tajima and J. M. Dawson, Phys. Rev. Lett. 43, 267 (1979).

[2] C. G. R. Geddes, C. Toth, J. van Tilborg, E. Esarey, C. B. Schroeder, D. Bruhwiler, C. Nieter, J. Cary, and W. P. Leemans, Nature (London) 431, 538 (2004).

[3] J. Faure, Y. Glinec, A. Pukhov, S. Kiselev, S. Gordienko, E. Lefebvre, J.-P. Rousseau, F. Burgy, and V. Malka, Nature (London) 431, 541 (2004).

[4] S. P. D. Mangles, C. D. Murphy, Z. Najmudin, A. G. R. Thomas, J. L. Collier, A. E. Dangor, E. J. Divall, P. S. Foster, J. G. Gallacher, C. J. Hooker, D. A. Jaroszynski, A. J. Langley, W. B. Mori, P. A. Norreys, F. S. Tsung,
R. Viskup, B. R. Walton, and K. Krushelnick, Nature (London) 431, 535 (2004).

[5] D. J. Spence, A. Butler, and S. M. Hooker, J. Phys. B 34, 4103 (2001).

[6] B. Cros, C. Courtois, G. Matthieussent, A. Di Bernardo, D. Batani, N. Andreev, and S. Kuznetsov, Phys. Rev. E 65, 026405 (2002).

[7] C. B. Schroeder, E. Esarey, C. G. R. Geddes, C. Benedetti, and W. P. Leemans, Phys. Rev. ST Accel. Beams 13, 101301 (2010).

[8] F. Wojda, K. Cassou, G. Genoud, M. Burza, Y. Glinec, O. Lundh, A. Persson, G. Vieux, E. Brunetti, R. P. Shanks, D. Jaroszynski, N. E. Andreev, C.-G. Wahlström, and B. Cros, Phys. Rev. E 80, 066403 (2009).

[9] G. Genoud, K. Cassou, F. Wojda, H. Ferrari, C. Kamperidis, M. Burza, A. Persson, J. Uhlig, S. Kneip, S. Mangles, A. Lifschitz, B. Cros, and C.-G. Wahlström, Appl. Phys. B 105, 309 (2011).

[10] H. E. Ferrari, A. F. Lifschitz, and B. Cros, Plasma Phys. Controlled Fusion 53, 014005 (2011).

[11] J. Osterhoff, A. Popp, Z. Major, B. Marx, T. P. Rowlands-Rees, M. Fuchs, M. Geissler, R. Hörlein, B. Hidding, S. Becker, E. A. Peralta, U. Schramm, F. Grüner, D. Habs, F. Krausz, S. M. Hooker, and S. Karsch, Phys. Rev. Lett. 101, 085002 (2008).

[12] G. Genoud, F. Wojda, M. Burza, A. Persson, and C.-G. Wahlstrom, Rev. Sci. Instrum. 82, 033102 (2011).

[13] J. Ju and B. Cros, J. Appl. Phys. 112, 113102 (2012).

[14] A. Buck, K. Zeil, A. Popp, K. Schmid, A. Jochmann, S. D. Kraft, B. Hidding, T. Kudyakov, C. M. S. Sears, L. Veisz, S. Karsch, J. Pawelke, R. Sauerbrey, T. Cowan, F. Krausz, and U. Schramm, Rev. Sci. Instrum. 81, 033301 (2010).

[15] P. Sprangle, C.-M. Tang, and E. Esarey, IEEE Trans. Plasma Sci. 15, 145 (1987).

[16] G.-Z. Sun, E. Ott, Y. C. Lee, and P. Guzdar, Phys. Fluids 30, 526 (1987)

[17] J. Ju, K. Svensson, H. Ferrari, A. Dopp, G. Genoud, F. Wojda, M. Burza, A. Persson, O. Lundh, C.-G. Wahlstrom, and B. Cros, Phys. Plasmas 20, 083106 (2013).

[18] S. P. D. Mangles, G. Genoud, M. S. Bloom, M. Burza, Z. Najmudin, A. Persson, K. Svensson, A. G. R. Thomas, and C.-G. Wahlström, Phys. Rev. ST Accel. Beams 15, 011302 (2012).

[19] S. Corde, C. Thaury, A. Lifschitz, G. Lambert, K. Ta Phuoc, X. Davoine, R. Lehe, D. Douillet, A. Rousse, and V. Malka, Nat. Commun. 4, 1501 (2013).

[20] S. Banerjee, S. Y. Kalmykov, N. D. Powers, G. Golovin, V. Ramanathan, N. J. Cunningham, K. J. Brown, S. Chen, I. Ghebregziabher, B. A. Shadwick, D. P. Umstadter, B. M. Cowan, D. L. Bruhwiler, A. Beck, and E. Lefebvre, Phys. Rev. ST Accel. Beams 16, 031302 (2013). 